\title{
Democratisation of AAC Symbol Design for People with Intellectual Disabilities
}

\author{
David Banes*1 and EA Draffan ${ }^{2}$ \\ ${ }^{1}$ University of Southampton, UK \\ ${ }^{2}$ Directors of Global Symbols CIC, UK
}

Submission: October 05, 2020; Published: October 08, 2020

*Corresponding author: David Banes, University of Southampton, UK

\begin{abstract}
The selection and design of AAC symbols has often been in the domain of those working with potential users rather than led by their paricipation. The paper reviews the work of a team who decided to democratise the process and use inclusive co-design methodologies. The lessons learnt were important and shared to the extent that a three year projec has entered its fifth year supported by organisations interested in global reach and open access. AAC users with intellectual disabilites were fully engaged in decision making for the design of symbols developed both as additions and newly designed open licenced symbol sets. This encouraged design that reflectd the users' perceptions of cultural and social settings, increasing a sense of famiarity and ownwership.as representative of the countries in which they have been designed. Users also had their own core vocabularies and orthography which highlighted the need for more research into the differences that are occurring between multilingual symbol sets and the need for more work in this area.
\end{abstract}

Keywords: Communication; AAC; democratization; co-design; symbols; culture; self-advocacy; open license; speech; language

\section{Introduction}

Familiarity and confidence are vital pre-requisites for successful implementation and use of a symbol set for an augmentative and alternative communication (AAC) user with intellectual disabilities [1]. Choosing such a suitable symbol set and the individual symbols within it can be difficult and at times frustrating. Traditionally AAC users have had a degree of engagement in such choices, but as symbol sets expand and encompass a wider range of languages and cultures, the right of users to determine both the design and core vocabulary becomes increasingly vital.

Global comparative word lists for the use of vocabulary suitable to developing symbol sets may not be readily available in some languages and it has been suggested that professionals may not necessarily be the best people to make such selections [2]. However, discussions around the use of participatory co-design that is inclusive and allows a wider audience to be included in the development of AAC and assistive technology systems is an achievable goal, where "multiple participants in social settings, rather than subjects in carefully controlled laboratories are involved" [3]. This concept has been embraced by the Global Symbols team.
Global Symbols, a team of AAC professionals with experience across the world, took a strategic decision to increasingly devolve decision making regarding symbol set choices and the creation of additional open licensed symbol sets to AAC users thanks to active support from their families and carers, alongside professionals. Furthermore, enabling participation in symbol design allowed for improved adaptations for those with intellectual disabilities, such as whole-body representations and realistic depictions of certain buildings and types of food. Participants were also involved the building of a list of frequently used words and phrases in local languages that supported both communication and literacy. Throughout each project in whatever country, despite the need for face to face discussions along with home and school visits, technology was found to be critical in achieving the goal of inclusive design. It allowed for independent voting to take place and the gathering of reactions as to why a symbol was or was not acceptable as users indicated choices.

Democratization has been defined as "the action of making something accessible to everyone" 1 . In this case the term has been linked to the work that went into enabling those using AAC devices access to the Global Symbols voting system. There was a need to 
ensure adequate and effective support from families, carers and professionals, with clearly defined roles and constraints. Anyone could participate in the process and potential users were identified through support organizations who contacted clients' families to suggest participation. Those willing to participate could choose the amount of time they wished to spend on the project and could leave at any time.

In an early use of the voting system the research indicated that, in order to reduce barriers to participation, an online symbol management system needed careful trialing with therapists and teachers as well as AAC users who also had mobility, dexterity and cognitive disabilities. Bespoke grids for communication software were developed that used the newly designed symbols and their labels. The feedback suggested that users preferred the choices to be presented in a form similar to a flashcard with large submit /previous/next buttons and a Likert scale that was set at 1-5 acceptance levels where 5 was completely acceptable with a comment box in a free text field. For some users it became clear that a simpler interface was required and a 1-3 scale or a simpler 'thumbs up' and 'thumbs down' symbol version was offered. Later versions of the voting system allowed for independent uploading of symbols and the setting up of multiple surveys with options to save voting sessions and collect any number of individual sessions, so that individuals were not overwhelmed by the number of symbols that needed to be viewed. This was particularly important for those with intellectual disabilities, whereas some therapists preferred to see multiple symbols and quickly go through the process.

\section{Discussion}

Success is best defined by the number of individuals who use the symbols to communicate and engage with content. Results suggest that the ideas have been successful in reaching and engaging participants with a wide range of abilities, needs, languages and cultural backgrounds. In Qatar, users prioritized additional, but critical symbols that could be used to facilitate inclusion in Islamic prayer, a collective act of worship. In the video "Share and Believe, A Symbolic Journey" Mohammed is seen praying using the Tawasol symbol set and his brother comments "I am so happy because Mohammed is able to pray with me using the prayer symbols on his Eye Gaze system. Knowing that he is excused from praying, but I am happy to see him trying to pray." In Pakistan it was found that users preferred tablets to laptops when voting and nationally dressed individuals and in Croatia videos were made with children successfully using the symbols on AAC apps after six months.

Huer (1997) reported that "observations of communication across cultures reveal that nonsymbolic as well as symbolic forms of communication are culturally dependent" [4] and in 2000 stated that 'participants' cultural/linguistic experiences may be significant elements to consider when selecting graphic symbols and when teaching consumers to represent meaning through them." [5] Personal preferences need to be taken into account, but by devolving decision making through a user-friendly voting system, decisions can be made by those involved with AAC directly, especially when thinking about cultural, social, environmental and religious suitability. The choices made by those involved in the process can inform localization symbol design principles and support future guidance for others working with symbols in other cultures and social settings and environments across the world.

Global Symbols now hosts over 40,500 freely available symbols and many can be adapted to suit individual preferences, depending on the creative commons license. New symbol sets are available in Urdu, Arabic, Serbian (Latin and Cyrillic script) and Croatian languages and can used alongside other sets where generic symbols can be found, such as certain animals etc. Some of the sets are available within AAC apps and all can be used in chart print outs and posters. Translations are on offer and adaptations are encouraged. To date they have reached 50 countries.

Whilst a democratic model of participation with symbol users has been established, further research and development is underway to create tools to make the graphic design process easier, founded upon crowdsourcing methods of symbol acceptance. It is hoped these endeavors will encourage those working in this area to consider adapting more symbols to suit adults with intellectual impairments, as there are very few freely available AAC symbols that truly represent their needs.

\section{Conclusion}

There remains a need to increase knowledge and use of participatory research and co-design that has been enabled through accessible and inclusive technologies to allow users to participate readily and easily. The information gathered in this process suggests that there is also a need to consider the content of core and fringe vocabularies across language communities where "norms" of communication and culture may vary considerably.

User defined core vocabularies are fundamental to the design of communication systems and both localization and personalization of symbol-based communication systems is essential and should be led by users with appropriate assistance. Impersonal language translation options inevitably fail to recognize the obvious and subtle differences between the wide range of social, linguistic, environment and religious nuances experienced by all AAC users in their own surroundings.

In seeking to engage fully with stakeholders to create a symbol selection process that has been attuned to cultural need and nuance, the approach discussed has moved from involving users and their supporters as participants, to transferring decision making to them collectively. The model is described as a democratization of that process reflecting a shift from consultation to decision making. This paradigm shift is both influenced by and reflects concepts of crowd sourcing of design as suggested by Brabham who refers to this as an "online, distributed problem- 
solving and production model" [6]. Whilst this model has been well documented in business and public sector decision making, it has been slow to be adopted in the assistive technology and AAC communities where final decisions have tended to remain the domain of the researcher or entrepreneur.

\section{Conflict of Interest}

EA Draffan and David Banes are co-founders and directors of Global Symbols CIC a not for profit company operating from the United Kingdom.

\section{References}

1. Wilkinson KM, Mcllvane WJ (2013) Perceptual factors influence visual search for meaningful symbols in individuals with intellectual disabilities and Down syndrome or autism spectrum disorders American Journal on Intellectual and Developmental Disabilities 118(5): 353-364.
2. Balandin S, Iacono T (1998) A few well-chosen words. Augmentative and Alternative Communication 14(3): 147-161.

3. Pullin G, Treviranus J, Patel R, Higginbotham J (2017) Designing interaction, voice, and inclusion in AAC research. Augmentative and Alternative Communication 33(3): 139-148.

4. Huer MB (1997) Culturally inclusive assessments for children using augmentative and alternative communication (AAC). Journal of Children's Communication Development 19 (1): 23-24.

5. Huer MB (2000) Examining perceptions of graphic symbols across cultures: Preliminary study of the impact of culture/ethnicity. Augmentative and Alternative Communication 16(3): 180-185.

6. Brabham DC (2008) Crowdsourcing as a Model for Problem Solving: An Introduction and Cases Convergence: The International Journal of Research into New Media Technologies 14 (1): 75-90.

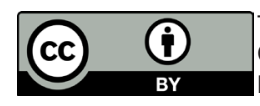

This work is licensed under Creative Commons Attribution 4.0 License DOI: 10.19080/GJIDD.2020.07.555704

\section{Your next submission with Juniper Publishers will reach you the below assets}

- Quality Editorial service

- Swift Peer Review

- Reprints availability

- E-prints Service

- Manuscript Podcast for convenient understanding

- Global attainment for your research

- Manuscript accessibility in different formats ( Pdf, E-pub, Full Text, Audio)

- Unceasing customer service

Track the below URL for one-step submission https://juniperpublishers.com/online-submission.php 